Geriatric medicine

\section{An elderly man with chest pain, shortness of breath, and constipation}

\section{A A Fisher, M W Davis}

Answers on $p 183$.

$A^{n}$ 81 year old man was admitted to hospital with fractured neck of his right femur. From the 1lth day after surgery he developed recurrent episodes of retrosternal and right lower chest pain associated with shortness of breath, sweating, nausea, and hiccup; the pain was aggravated by deep breathing. He had four such episodes in three days while walking with a frame. On each occasion he was given glyceryl trinitrate ( $600 \mu \mathrm{g}$ sublingually). The pain lasted from 30 minutes to two hours and was relieved in the supine position. He was constipated for five days. His past medical history included ischaemic heart disease with coronary artery bypass three years earlier but no angina since, stomach surgery for bleeding peptic ulcer ( 10 years previously), bilatera total knee replacements (two years previously), bladder cancer, and a long history of constipation. He never smoked and used alcohol only occasionally. His regular medications included trandolapril (1 mg daily), metoprolol (50 mg daily), ranitidine (150 mg twice a day), calcitriol $(0.25 \mu \mathrm{g}$ twice a day), coloxyl with senna (two tablets daily), and enoxaparin sodium (40 mg daily). For pain control he was taking paracetamol ( $1 \mathrm{~g}$ four times a day) and oxycodone hydrochloride (2.5-5 mg 3-6 times a day as needed) since surgery.

On physical examination (during these four episodes) he was afebrile with a respiratory rate of $16-20$ breaths $/ \mathrm{min}$, heart rate of 90-100 beats/min, blood pressure ranging from $126 / 80$ to $140 / 84$ $\mathrm{mm} \mathrm{Hg}$, and oxygen saturation of $83 \%-$
$88 \%$ on air and $94 \%-96 \%$ on $2-4$ litres of oxygen. There were no heart murmurs nor signs of peripheral oedema. The breath sounds were decreased at the right base compared with the left, and there were a few bibasal inspiratory crackles but no pleural rub detected. The abdomen was mildly distended, tympanitic with mild to moderate tenderness and increased bowel sounds over the right upper quadrant, but no guarding nor rebound tenderness were noted. There was no hepatosplenomegaly. Serial electrocardiograms (ECGs) showed sinus rhythm, right bundle branch block, and left anterior hemiblock. Repeated studies of cardiac enzymes (creatinine kinase, troponin I), arterial blood gas measurements, D-dimer screen, serum electrolytes, urea, creatinine, and glucose as well as full blood count were within normal limits. Chest (see figs 1 and 2) and abdominal radiographs and a computed tomographic pulmonary angiogram were performed.

\section{QUESTIONS}

(1) What do the chest radiographs show?

(2) What important physical sign may have been missed?

(3) What is the differential diagnosis?

(4) What were the predisposing factors to this condition?

(5) What is the management of this condition?

Postgrad Med J 2003;79:180
Authors' affiliations

A A Fisher, M W Davis, Department of

Geriatric Medicine, Canberra Hospital and Canberra Clinical School of the University of

Sydney, Australia

Correspondence to: Dr Michael W Davis, Department of Geriatric Medicine, Canberra Hospital, PO Box 11 Woden ACT 2606 Australia; michaelw.davis@act.gov.au

Submitted 19 June 2002

Accepted 25 November 2002

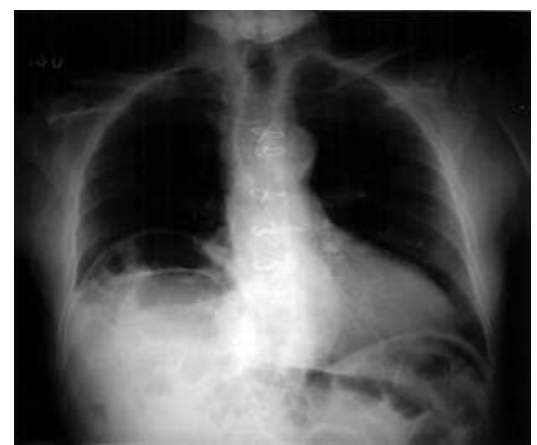

Figure 1 Chest radiograph: posteroanterior view.

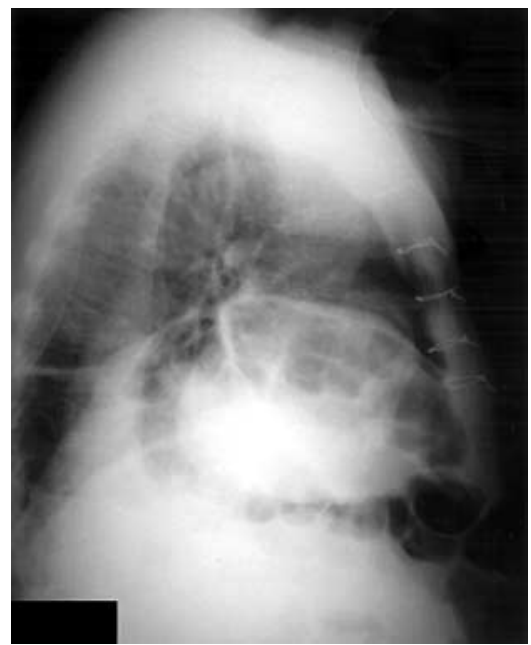

Figure 2 Chest radiograph: lateral view. 